PROCEEDINGS OF THE

AMERICAN MATHEMATICAL SOCIETY

Volume 137, Number 9, September 2009, Pages 3077-3090

S 0002-9939(09)09852-9

Article electronically published on March 18, 2009

\title{
QUADRATIC RATIONAL MAPS LACKING PERIOD 2 ORBITS
}

\author{
RIKA HAGIHARA
}

(Communicated by Jane M. Hawkins)

\begin{abstract}
We study dynamical properties of a parameterized family of quadratic rational maps, all of whose members lack period 2 orbits. We classify regions in the parameter space of the family according to the behavior of marked critical points. We characterize the parameter space by comparing it with the Mandelbrot set.
\end{abstract}

\section{INTRODUCTION}

In the theory of iteration of rational maps, periodic points play important roles. Every rational map has infinitely many periodic points (Beardon [2]); however, some rational maps fail to have periodic points of a certain period. Baker 1 proved that if a polynomial $P$ of degree at least 2 has no periodic points of period $n$, then $n=2$ and $P$ is conformally conjugate to $z \mapsto z^{2}-z$. As for rational maps, he proved the following.

Theorem 1.1 ([1]). Let $R$ be a rational map of degree $d$, where $d \geq 2$. If $R$ has no periodic points of period $n$, then the pair $(d, n)$ is either $(2,2),(2,3),(3,2)$ or $(4,2)$. Such $R$ exists for each pair $(d, n)$.

Kisaka 9 listed all possible forms of such exceptional rational maps. The author [6] gave a complete proof of the classification.

The $(2,2)$ case is conformally conjugate to the parameterized family of rational maps $R_{a}(z)=\frac{z^{2}-z}{a z+1}$, where $a \in \mathbb{C} \backslash\{-1\}$. Recall that every quadratic rational map has two distinct critical points (Milnor [13]). Members of the family $\left\{R_{a}\right\}$ can be characterized as having a parabolic fixed point with multiplier -1 and one immediate basin. Hence for each parameter $a$ one of the critical points of $R_{a}$ must be associated to the parabolic fixed point. The main purpose of this paper is to demonstrate the existence of a unique critical point associated to this parabolic fixed point. Consequently, there is a "free" critical point. Since, depending on the parameter value $a$, the map $R_{a}$ has an attracting cycle, we would expect to see a copy of the Mandelbrot set in the parameter space of $\left\{R_{a}\right\}$ arising from the bifurcation of this free critical point. The parameter space of $\left\{R_{a}\right\}$ in $[-3,3] \times$ $[-3 i, 3 i]$ is shown in Figure 1, A holomorphic copy of the figure appears in [13].

This paper is organized as follows. In Section 2 we collect preliminary results about $R_{a}$ and use them to reduce the parameter space of $\left\{R_{a}\right\}$ by conjugacy.

Received by the editors October 23, 2008, and, in revised form, December 8, 2008.

2000 Mathematics Subject Classification. Primary 37F45; Secondary 30D05, 37F10.

Key words and phrases. Complex dynamics, parabolic, critical points.

(C)2009 American Mathematical Society 


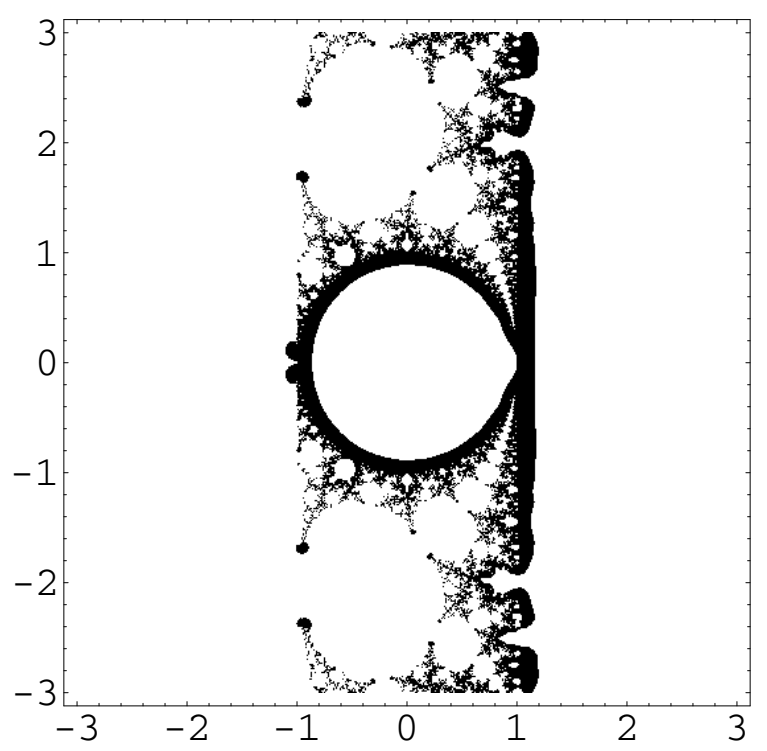

FIGURE 1. The parameter space of $\left\{R_{a}\right\}$.

In Section 3 we classify regions of the parameter space of $\left\{R_{a}\right\}$ according to the behavior of marked critical points. The main theorem asserts that we can specify a unique critical point associated to the parabolic fixed point of $R_{a}$. We further prove interesting dynamics that $R_{a}$ exhibits for certain parameter values. In Section 4 we compare the characteristics of the Mandelbrot set and the parameter space of $\left\{R_{a}\right\}$. The computer algorithm used for producing the parameter space picture is explained in the Appendix.

Much work has been done on the dynamics of quadratic rational maps. Milnor [13, 14] and Rees [15] worked on the moduli space of quadratic rational maps quite generally. Specific slices of the moduli space have also been studied by Hawkins [7] and Milnor [13], with the latter work including parabolic ones. Results in this article provide proofs of some of the observations in [13.

This paper is based on the author's dissertation research at the University of North Carolina at Chapel Hill under the supervision of Dr. Jane Hawkins. Some computer programs used are edited versions of Maple and Mathematica programs first written by Dr. Lorelei Koss.

\section{Reduction of the PARAmeter SPACE}

We first reduce the parameter space of $\left\{R_{a}\right\}$ by conformal conjugacy and then identify points of the reduced parameter space by symmetry. We define the equivalence relation among parameters by $a \sim a^{\prime}$ if and only if the corresponding maps $R_{a}$ and $R_{a^{\prime}}$ are conformally conjugate. With it we form a reduced set $\mathcal{R}_{a}$ by taking a representative from each equivalence class.

The fixed points of $R_{a}(z)=\frac{z^{2}-z}{a z+1}$ are $0, \infty$, and $\frac{2}{1-a}$ with multipliers $-1, a$, and $\frac{3-a}{1+a}$, respectively. Hence $R_{a}$ and $R_{a^{\prime}}$ are conformally conjugate if and only if $a^{\prime}=a$ or $a^{\prime}=\frac{3-a}{1+a}([2])$. Set $f(a)=\frac{3-a}{1+a}$. 


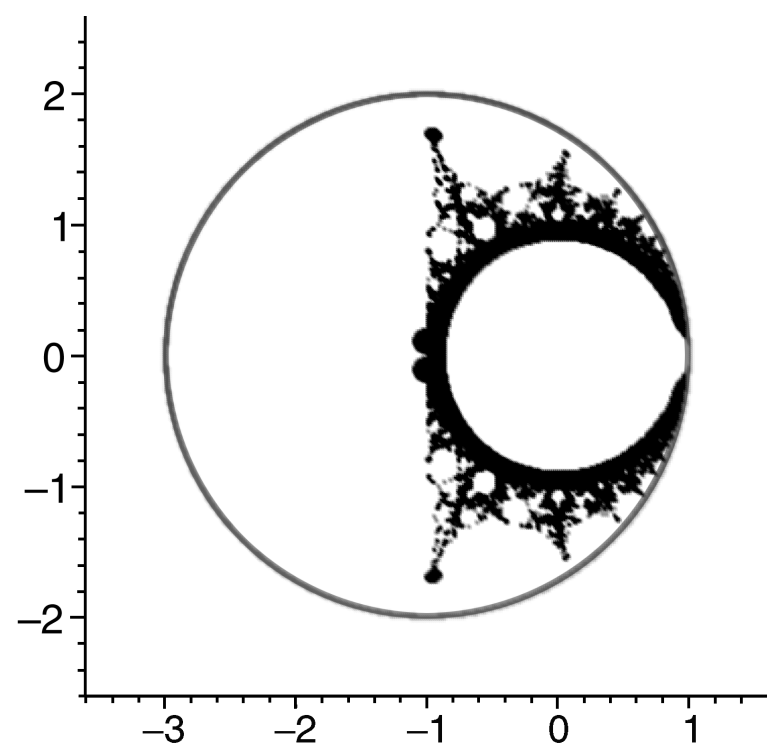

FiguRe 2. $\mathcal{R}_{a}$.

Lemma 2.1. The map $f$ satisfies the following.

(i) $a \sim f(a)$.

(ii) The inverse of $f$ is itself.

(iii) The function $f$ maps the circle $C=\{a \in \mathbb{C}:|a+1|=2\}$ onto itself.

Proof. The first two results follow from the definition of $f$ (see [9]). For (iii) an easy calculation shows that $f\left(-1+2 e^{i \theta}\right)=-1+2 e^{-i \theta}$ for $\theta \in \mathbb{R}$.

Theorem 2.2. A reduced set $\mathcal{R}_{a}$ can be taken as

$$
\mathcal{R}_{a}=\{a \in \mathbb{C}: 0<|a+1|<2\} \cup\{a \in \mathbb{C}:|a+1|=2,0 \leq \operatorname{Im}(a)\} .
$$

Proof. Note that $f:\{a \in \mathbb{C}: 0<|a+1|<2\} \rightarrow\{a \in \mathbb{C}: 2<|a+1|\}$ is a bijection. The proof of Lemma 2.1 shows that complex conjugates on $C=\{a \in \mathbb{C}:|a+1|=2\}$ can be identified with each other.

The set $\mathcal{R}_{a}$ is in the reduced form under conformal conjugacy. It is the region inside and the upper half of the circle in Figure 2. Next, we identify points of $\mathcal{R}_{a}$ using symmetry.

Lemma 2.3. For $\varphi(z)=\bar{z}$, we have $\varphi \circ R_{\bar{a}} \circ \varphi^{-1}=R_{a}$.

This implies that although $R_{a}$ and $R_{\bar{a}}$ are not conformally conjugate, their dynamics are symmetric about the real line, and thus the reduced set $\mathcal{R}_{a}$ has symmetry about the real line. Therefore, it suffices to use parameters in

$$
\mathcal{R}_{a}^{\mathrm{sym}}=\{a \in \mathbb{C}: 0<|a+1| \leq 2,0 \leq \operatorname{Im}(a)\}
$$

to study how the dynamics of $R_{a}$ depend on the parameter $a$. 


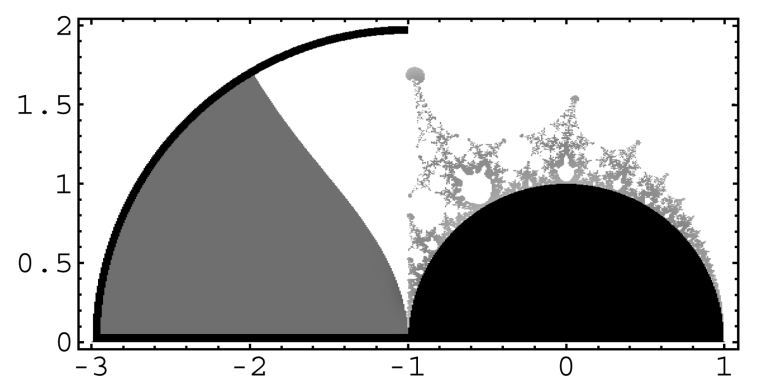

Figure $3 . A_{1}, A_{2}, A_{3}$, and $A_{4}$ in $\mathcal{R}_{a}^{\mathrm{sym}}$.

\section{The ROLES OF CRITICAL POINTS}

Each map $R_{a}(z)=\frac{z^{2}-z}{a z+1}$ has two distinct critical points. By construction $R_{a}$ has a parabolic fixed point 0 with one immediate basin consisting of two disjoint Fatou components. Hence for each parameter $a$ one of the critical points of $R_{a}$ must be associated to 0 . Theorem 3.1 asserts that for much of the reduced set $\mathcal{R}_{a}$ a unique critical point is associated to 0 .

Theorem 3.1. The orbit of the critical point $c_{1}=\frac{-1+\sqrt{1+a}}{a}$ of $R_{a}$ converges to the parabolic fixed point 0 for each parameter $a$ in the following sets:

$$
\begin{aligned}
& A_{1}=\left\{a \in \mathcal{R}_{a}:|a|<1\right\} \\
& A_{2}=\left\{a \in \mathcal{R}_{a}:-3 \leq a<-1\right\} \\
& A_{3}=\left\{a \in \mathcal{R}_{a}: \operatorname{Re}\left(a+\frac{4}{a+1}\right)<-3\right\} \\
& A_{4}=\left\{a \in \mathcal{R}_{a}:|a+1|=2,-3 \leq \operatorname{Re}(a)<-1\right\} .
\end{aligned}
$$

For parameters in $A_{1}$, the orbit of the critical point $c_{2}=\frac{-1-\sqrt{1+a}}{a}$ converges to the attracting fixed point $\infty$. For parameters in $A_{2}, A_{3}$, and $A_{4}$, the orbits of both critical points $c_{1}$ and $c_{2}$ converge to 0 . For parameters in $A_{4}$, the orbits of both critical points lie on the circle of center $\frac{2}{1-a}$ (another fixed point of $R_{a}$ ) and radius $\left|\frac{2}{1-a}\right|$.

The parameters in $\mathcal{R}_{a}^{\text {sym }}$ for which Theorem 3.1 holds are colored black (sets $\left.A_{1}, A_{2}, A_{4}\right)$ and gray $\left(A_{3}\right)$ in Figure 3. The proof is divided into cases which are proved as separate results.

Case 1. $A_{1}=\left\{a \in \mathcal{R}_{a}:|a|<1\right\}$. We use the following theorem.

Theorem 3.2 ([11]). Let $f_{\lambda}$ be a holomorphic family of rational maps parameterized by a connected complex manifold $\Lambda$, and let $x$ be a point in $\Lambda$. Suppose that $c_{i}: \Lambda \rightarrow \widehat{\mathbb{C}}$ are holomorphic maps parameterizing the critical points of $f_{\lambda}$. Then the following conditions are equivalent.

(i) The number of attracting cycles of $f_{\lambda}$ is locally constant at $x$.

(ii) For each $i$, the functions $\lambda \mapsto f_{\lambda}^{n}\left(c_{i}(\lambda)\right), n=0,1,2, \ldots$, form a normal family at $x$.

Proof for Case 1. For a parameter $a$ in $A_{1}=\mathbb{D}, R_{a}$ has an attracting fixed point $\infty$ with multiplier $a$ and a parabolic fixed point 0 . Since $R_{a}$ has two critical points 
$c_{1}$ and $c_{2}$, Theorem 3.2 implies that for each $i$ the family $\left\{\left(R_{a}\right)^{n}\left(c_{i}\right)\right\}_{n=0}^{\infty}$ converges normally at $a$ to either 0 or $\infty$. For $a=0$ the critical point $c_{2}$ itself is a superattracting fixed point $\infty$. Hence for each $a \in A_{1}$ the critical point $c_{1}$ is associated to 0 , and $c_{2}$ to $\infty$.

Case 2. $A_{2}=\left\{a \in \mathcal{R}_{a}:-3 \leq a<-1\right\}$.

Proof. The critical points $c_{1}$ and $c_{2}$ of $R_{a}$ are complex conjugates, and so are their corresponding iterates under $R_{a}$. Hence the orbits of both critical points converge to the parabolic fixed point 0 .

Case 3. $A_{3}=\left\{a \in \mathcal{R}_{a}: \operatorname{Re}\left(a+\frac{4}{a+1}\right)<-3\right\}$. We use results of Bergweiler [3] and Buff and Epstein [5] on the number of critical points in the immediate basins of a parabolic fixed point.

Lemma 3.3 ([3], [5]). Let $R$ be a rational map having a fixed point $\alpha$ with multiplier $e^{2 \pi i \frac{p}{q}}$. Then there exists an integer $k \geq 1$ called the parabolic multiplicity of $\alpha$ and a local holomorphic coordinate $\varphi$ defined near $\alpha$ such that $\varphi(\alpha)=0$ and

$$
\left(\varphi \circ R \circ \varphi^{-1}\right)(z)=e^{2 \pi i \frac{p}{q}} z\left(1+z^{q k}+\beta z^{2 q k}\right)+\mathrm{O}\left(|z|^{2 q k+2}\right) .
$$

A holomorphic change of coordinates leaves $\beta$ invariant.

Define the résidu iteratif of $R$ at $\alpha$ by $\operatorname{résit}(R, \alpha)=\frac{q k+1}{2}-\beta$.

Theorem 3.4 ([3], [5]). Let $R$ be a rational map having a fixed point $\alpha$ with multiplier $e^{2 \pi i \frac{p}{q}}$ and parabolic multiplicity $k$. If the union of the immediate basins of $\alpha$ contains exactly $k$ simple critical points of $R$, then $\operatorname{Re}\left(\operatorname{résit}\left(R^{q}, \alpha\right)\right) \geq \frac{q k}{4}$. In particular, when $\operatorname{Re}\left(\right.$ résit $\left.\left(R^{q}, \alpha\right)\right)<\frac{q k}{4}$, the union of the immediate basins of $\alpha$ contains at least $k+1$ critical points of $R$, counting multiplicity.

Proof for Case 3. In the setting of $R_{a}{ }^{2}$ we have $\alpha=0, p=q=1$, and $k=2$. Write $a=\lambda e^{i \theta}-1$, where $0<\lambda \leq 2$ and $\frac{\pi}{2}<\theta \leq \pi$. Conjugate $R_{a}{ }^{2}$ first by $\varphi_{1}(z)=i \sqrt{2 \lambda} e^{i \frac{\theta}{2}} z$ and then by $\varphi_{2}(z)=z\left(1+\frac{i \sqrt{\lambda} e^{i \frac{\theta}{2}}}{2 \sqrt{2}} z\right)$ so that

$$
\left(\left(\varphi_{2} \circ \varphi_{1}\right) \circ\left(R_{a}^{2}\right) \circ\left(\varphi_{2} \circ \varphi_{1}\right)^{-1}\right)(z)=z+z^{3}+\frac{1}{8}\left(5-a-\frac{4}{a+1}\right) z^{5}+\mathrm{O}\left(z^{6}\right) .
$$

Hence $\beta=\frac{1}{8}\left(5-a-\frac{4}{a+1}\right)$ for $R_{a}^{2}$ and

$$
\text { résit }\left(R_{a}^{2}, 0\right)=\frac{1 \cdot 2+1}{2}-\frac{1}{8}\left(5-a-\frac{4}{a+1}\right)=\frac{1}{8}\left(7+a+\frac{4}{a+1}\right) .
$$

For $R_{a}$ we have $\alpha=0, p=1, q=2$, and $k=1$. The second part of Theorem 3.4 implies that when $\operatorname{Re}\left(\operatorname{résit}\left(R_{a}{ }^{2}, 0\right)\right)<\frac{2 \cdot 1}{4}=\frac{1}{2}$, the immediate basin of the parabolic fixed point 0 contains both critical points $c_{1}$ and $c_{2}$. This happens when $\operatorname{Re}\left(\operatorname{résit}\left(R_{a}{ }^{2}, 0\right)\right)=\operatorname{Re}\left(\frac{1}{8}\left(7+a+\frac{4}{a+1}\right)\right)<\frac{1}{2}$, i.e. when $\operatorname{Re}\left(a+\frac{4}{a+1}\right)<-3$.

Case 4. $A_{4}=\left\{a \in \mathcal{R}_{a}:|a+1|=2,-3 \leq \operatorname{Re}(a)<-1\right\}$. The main steps of the proof are as follows. We first show that both critical points $c_{1}$ and $c_{2}$ as well as the fixed point 0 of $R_{a}$ lie on the circle $C_{\theta}$ in $\mathbb{C}$ of center $\frac{2}{1-a}$ (another fixed point of $R_{a}$ ). This circle is shown to be forward invariant under $R_{a}$. We next consider the relative location of the critical points and some of their iterative images under $R_{a}$. We then form on $C_{\theta}$ a simple path from each critical point to 0 . With the information on the location of key points, we proceed to show that the successive images of the paths under $R_{a}$ shrink to 0 . We now provide the details of the proof. 
We first prove a result on the location of $c_{1}$ and $c_{2}$ in a more general setting. For a parameter $a$ in $\mathcal{R}_{a}^{\text {sym }}=\{a \in \mathbb{C}: 0<|a+1| \leq 2,0 \leq \operatorname{Im}(a)\}$, write $a=\lambda e^{i \theta}-1$, where $0<\lambda \leq 2$ and $0 \leq \theta \leq \pi$. With this notation the critical points of $R_{a}$ are $c_{1}=\frac{1}{1+\sqrt{\lambda} e^{i \frac{\theta}{2}}}$ and $c_{2}=\frac{1}{1-\sqrt{\lambda} e^{i \frac{\theta}{2}}}$. Define a circle $C_{\theta}$ in $\mathbb{C}$ as having center $c=\frac{1}{1-e^{i \theta}}$ and radius $|c|$, so that $C_{\theta}$ passes through the fixed point 0 of $R_{a}$.

Proposition 3.5. For every $a=\lambda e^{i \theta}-1$, where $0<\lambda \leq 2$ and $0<\theta \leq \pi$, both critical points $c_{1}$ and $c_{2}$ of $R_{a}$ lie on $C_{\theta}$.

Proof. Using the trigonometric expressions for the center $c=\frac{1}{2}+\frac{i}{2} \cdot \frac{\cos \frac{\theta}{2}}{\sin \frac{\theta}{2}}$ of $C_{\theta}$ and the critical point $c_{1}=\frac{\cos \frac{\theta}{2}+\frac{1}{\sqrt{\lambda}}-i \sin \frac{\theta}{2}}{\frac{\lambda+1}{\sqrt{\lambda}}+2 \cos \frac{\theta}{2}}$ of $R_{a}$, we calculate that $|c|^{2}=\left|c-c_{1}\right|^{2}=$ $\frac{1}{4 \sin ^{2} \frac{\theta}{2}}$. A calculation for $c_{2}$ is similar.

Corollary 3.6. In the setting of Proposition 3.5 , we have $\operatorname{Re}(c)=\frac{1}{2}$ and $C_{\theta} \cap \mathbb{R}=$ $\{0,1\}$.

Proof. The circle $C_{\theta}$ has a center whose real coordinate is $\frac{1}{2}$, and $C_{\theta}$ passes through 0 . By symmetry $C_{\theta}$ intersects with the real axis at 1 . Indeed, the point 1 is a preimage of 0 .

Remarks 3.7. When $\theta=0$, the point $c=\frac{1}{1-e^{i \theta}}$ is $\infty$ and the critical points $c_{1}$ and $c_{2}$ have real values. Hence $c, c_{1}$, and $c_{2}$ can be viewed as lying on the "extended" circle $C_{\mathbb{R}}=\{z \in \mathbb{R}\} \cup\{\infty\}$ in $\widehat{\mathbb{C}}$.

Next, we restrict to parameters on a part of the semi-circular boundary of $\mathcal{R}_{a}^{\mathrm{sym}}$.

Lemma 3.8. For every $a=2 e^{i \theta}-1$, where $0<\theta \leq \pi$, the following hold.

(i) The center $c$ of $C_{\theta}$ coincides with the fixed point $\frac{2}{1-a}$ of $R_{a}$.

(ii) The circle $C_{\theta}$ is forward invariant under $R_{a}$.

Proof. When $\lambda=2$ (so that $|a+1|=2)$ and $\theta \neq 0(a \neq 1)$, an easy calculation shows that the center $\frac{1}{1-e^{i \theta}}$ of $C_{\theta}$ coincides with the fixed point $\frac{2}{1-a}$ of $R_{a}$ and that $C_{\theta}$ has radius $|c|=\frac{-i e^{\frac{i \theta}{2}}}{1-e^{i \theta}}$. Write an arbitrary point $p$ on $C_{\theta}$ as

$$
p=\frac{1}{1-e^{i \theta}}+\frac{-i e^{\frac{i \theta}{2}}}{1-e^{i \theta}} \cdot e^{i \alpha},
$$

where $\alpha \in \mathbb{R}$. Then

$$
R_{a}(p)=-\frac{\left(i+e^{\frac{i}{2}(2 \alpha+\theta)}\right)\left(e^{\frac{i \theta}{2}}-i e^{i \alpha}\right)}{\left(-1+e^{i \theta}\right)\left(-e^{i \alpha}+i e^{\frac{i \theta}{2}}+2 e^{i(\alpha+\theta)}\right)} .
$$

We need to check the distance between $R_{a}(p)$ and the center $c$ of $C_{\theta}$ :

$$
R_{a}(p)-c=\frac{e^{i \alpha}\left(e^{i \theta}+i e^{\frac{i}{2}(2 \alpha+\theta)}-2\right)}{\left(-1+e^{i \theta}\right)\left(-e^{i \alpha}+i e^{\frac{i \theta}{2}}+2 e^{i(\alpha+\theta)}\right)} .
$$

In order to see that $R_{a}(p)-c$ has modulus $|c|=\frac{1}{\left|1-e^{i \theta}\right|}$, we multiply $R_{a}(p)-c$ by $-e^{-i(\alpha+\theta)}$ of unit modulus and obtain

$$
\frac{e^{i \alpha}\left(-e^{-i \alpha}-i e^{-\frac{i \theta}{2}}+2 e^{-i(\alpha+\theta)}\right)}{\left(-1+e^{i \theta}\right)\left(-e^{i \alpha}+i e^{\frac{i \theta}{2}}+2 e^{i(\alpha+\theta)}\right)} .
$$


The factors $-e^{-i \alpha}-i e^{-\frac{i \theta}{2}}+2 e^{-i(\alpha+\theta)}$ in the numerator and $-e^{i \alpha}+i e^{\frac{i \theta}{2}}+2 e^{i(\alpha+\theta)}$ in the denominator are complex conjugates. Since $\alpha \in \mathbb{R}$, the difference $R_{a}(p)-c$ has modulus $\left|\frac{e^{i \alpha}}{-1+e^{i \theta}}\right|=\frac{1}{\left|1-e^{i \theta}\right|}$. Therefore, a point on the circle $C_{\theta}$ is mapped to a point on the same circle.

For the rest of this section we restrict our attention to $A_{4}$ and assume that $a=2 e^{i \theta}-1$, where $\frac{\pi}{2}<\theta \leq \pi$. Let $\ell$ denote the line in $\mathbb{C}$ that passes through 0 and the center $c$ of the circle $C_{\theta}$. Then $\ell$ splits $C_{\theta}$ into two halves. Let $\ell^{\prime}$ be the tangent line to $C_{\theta}$ at 0 .

When $\theta=\pi$, the circle $C_{\theta}$ has center $\frac{1}{2}$ and radius $\frac{1}{2}$. So $\ell$ is the real axis and $\ell^{\prime}$ the imaginary axis in $\mathbb{C}$ : in fact, $\ell \cup\{\infty\}=J$ (Proposition 3.18) and $\ell^{\prime}$ is the axis of petals at 0 . The critical point $c_{1}$ and the image $R_{a}\left(c_{2}\right)$ of $c_{2}$ are on $C_{\theta}$ and on the same side along $\ell$, while $c_{2}$ and $R_{a}\left(c_{1}\right)$ are on $C_{\theta}$ and on the other side.

Note that $c, c_{1}, c_{2}, R_{a}\left(c_{1}\right)$, and $R_{a}\left(c_{2}\right)$ move continuously with respect to $\theta$. We use the notation $c_{1}(\theta)$, for example, to emphasize that $c_{1}$ is a continuous function of $\theta$. The next lemma shows that for $\theta \in\left(\frac{\pi}{2}, \pi\right), c_{1}$ and $R_{a}\left(c_{2}\right)$ stay on one side along $\ell$, while $c_{2}$ and $R_{a}\left(c_{1}\right)$ remain on the other.

Lemma 3.9. For $\theta \in\left(\frac{\pi}{2}, \pi\right)$, the following hold.

(i) Both $\left|c_{1}\right|$ and $\left|R_{a}\left(c_{1}\right)\right|$ are neither the diameter of the circle $C_{\theta}$ nor 0 .

(ii) Both $\left|c_{2}\right|$ and $\left|R_{a}\left(c_{2}\right)\right|$ are neither the diameter of the circle $C_{\theta}$ nor 0 .

Proof. (i) For $c_{1}=\frac{1}{1+\sqrt{2} e^{i \frac{\theta}{2}}}$, we have $\left|c_{1}\right|^{2}=\left|c_{1}\right|^{2}(\theta)=\frac{1}{3+2 \sqrt{2} \cos \frac{\theta}{2}}$, so $\left|c_{1}\right|^{2}$ and hence $\left|c_{1}\right|$ are monotone increasing on $\left(\frac{\pi}{2}, \pi\right)$. As for the diameter of $C_{\theta}$, it is $2|c|=\frac{1}{\sin \frac{\theta}{2}}$, which is monotone decreasing on $\left(\frac{\pi}{2}, \pi\right)$. We see that $\left|c_{1}\right|$ and $2|c|$ satisfy the inequality $0<\frac{1}{\sqrt{5}}=\left|c_{1}\right|\left(\frac{\pi}{2}\right)<\left|c_{1}\right|(\pi)=\frac{1}{\sqrt{3}}<1=2|c|(\pi)$. Thus $\left|c_{1}\right|$ is neither the diameter of $C_{\theta}$ nor 0 for $\theta \in\left(\frac{\pi}{2}, \pi\right)$. For $R_{a}\left(c_{1}\right)=\frac{-1}{\left(1+\sqrt{2} e^{i \frac{\theta}{2}}\right)^{2}}=-c_{1}^{2}$, the above inequality implies that $\left|R_{a}\left(c_{1}\right)\right|$ satisfies the claim.

(ii) For $c_{2}=\frac{1}{1-\sqrt{2} e^{i \frac{\theta}{2}}}$, we have $\left|c_{2}\right|^{2}=\frac{1}{3-2 \sqrt{2} \cos \frac{\theta}{2}}$, so $\left|c_{2}\right|^{2}$ and hence $\left|c_{2}\right|$ are monotone decreasing on $\left(\frac{\pi}{2}, \pi\right)$. Note that the inequality $0<\frac{1}{\sqrt{3}}=\left|c_{2}\right|(\pi)<$ $\left|c_{2}\right|\left(\frac{\pi}{2}\right)=1=2|c|(\pi)$ holds. As for $R_{a}\left(c_{2}\right)=\frac{-1}{\left(1-\sqrt{2} e^{i \frac{\theta}{2}}\right)^{2}}=-c_{2}^{2}$, we have $0<\frac{1}{3}=$ $\left|R_{a}\left(c_{2}\right)\right|(\pi)<\left|R_{a}\left(c_{2}\right)\right|\left(\frac{\pi}{2}\right)=1=2|c|(\pi)$. Hence both $\left|c_{2}\right|$ and $\left|R_{a}\left(c_{2}\right)\right|$ are neither $2|c|$ nor 0 for $\theta \in\left(\frac{\pi}{2}, \pi\right)$.

Remarks 3.10. The proof of Lemma 3.9 in particular shows that $0<\left|c_{1}\right|<1<2|c|$, $0<\left|c_{2}\right|<1$, and $0<\left|R_{a}\left(c_{2}\right)\right|<1$ for $\theta \in\left(\frac{\pi}{2}, \pi\right)$.

The following two lemmas compare the moduli of the critical points and some iterative images of $c_{1}$ under $R_{a}$.

Lemma 3.11. The inequality $\left|R_{a}\left(c_{1}\right)\right|<\left|c_{2}\right|$ holds for $\theta \in\left(\frac{\pi}{2}, \pi\right)$.

Proof. We have $\left|R_{a}\left(c_{1}\right)\right|=\frac{1}{3+2 \sqrt{2} \cos \frac{\theta}{2}}$ and $\left|c_{2}\right|^{2}=\frac{1}{3-2 \sqrt{2} \cos \frac{\theta}{2}}$. Hence for $\theta \in\left(\frac{\pi}{2}, \pi\right)$ the inequality $0<\left|R_{a}\left(c_{1}\right)\right|<\left|c_{2}\right|^{2}$ holds. Since $0<\left|c_{2}\right|<1$ by Remarks 3.10. it must be that $0<\left|R_{a}\left(c_{1}\right)\right|<\left|c_{2}\right|$ for $\theta \in\left(\frac{\pi}{2}, \pi\right)$.

Lemma 3.12. The inequality $\left|R_{a}^{2}\left(c_{1}\right)\right|<\left|c_{1}\right|$ holds for $\theta \in\left(\frac{\pi}{2}, \pi\right)$. 
Proof. With $R_{a}{ }^{2}\left(c_{1}\right)=\frac{1+\sqrt{2} e^{i \frac{\theta}{2}}+e^{i \theta}}{\left(1+\sqrt{2} e^{i \frac{\theta}{2}}\right)^{3}}$ we calculate

$$
\left|c_{1}\right|-\left|R_{a}^{2}\left(c_{1}\right)\right|=\frac{2\left|e^{i \theta}+\sqrt{2} e^{i \frac{\theta}{2}}+\frac{1}{2}\right|-\left|1+\sqrt{2} e^{i \frac{\theta}{2}}+e^{i \theta}\right|}{\left|1+\sqrt{2} e^{i \frac{\theta}{2}}\right|^{3}} .
$$

Set $F(\theta)=2\left|e^{i \theta}+\sqrt{2} e^{i \frac{\theta}{2}}+\frac{1}{2}\right|-\left|1+\sqrt{2} e^{i \frac{\theta}{2}}+e^{i \theta}\right|$. Then

$$
\begin{aligned}
F(\theta) & \geq 2\left(\left|e^{i \theta}+\sqrt{2} e^{i \frac{\theta}{2}}+1\right|-\frac{1}{2}\right)-\left|e^{i \theta}+\sqrt{2} e^{i \frac{\theta}{2}}+1\right| \\
& =\left|e^{i \theta}+\sqrt{2} e^{i \frac{\theta}{2}}+1\right|-1 \\
& \geq\left|e^{i \frac{\theta}{2}}+\frac{\sqrt{2}}{2}\right|^{2}-\frac{1}{2}-1=\left|e^{i \frac{\theta}{2}}+\frac{\sqrt{2}}{2}\right|^{2}-\frac{3}{2} .
\end{aligned}
$$

Note that $\left|e^{i \frac{\theta}{2}}+\frac{\sqrt{2}}{2}\right|^{2}=\frac{3}{2}+\sqrt{2} \cos \frac{\theta}{2}>\frac{3}{2}$ for $\theta \in\left(\frac{\pi}{2}, \pi\right)$. Hence $F>0$ and thus $\left|c_{1}\right|-\left|R_{a}^{2}\left(c_{1}\right)\right|>0$ for $\theta \in\left(\frac{\pi}{2}, \pi\right)$.

So far we have shown that for each parameter value $a$ in $A_{4}$ the fixed point 0 and the critical points $c_{1}, c_{2}$ of $R_{a}$ lie on the forward invariant circle $C_{\theta}$ of center $\frac{2}{1-a}$. We have also checked the relative location and moduli of the critical points and some of their iterative images under $R_{a}$.

We now construct simple paths on $C_{\theta}$ from critical points $c_{i}$ to 0 and show that their successive images under $R_{a}$ shrink to 0 . Cut $C_{\theta}$ at $c_{i}$ and 0 and form two arcs on $C_{\theta}$ whose end points are $c_{i}$ and 0 . Denote by $c_{i} 0$ the shorter of the two. Let $f_{c_{i}}:[0,1] \rightarrow C_{\theta}$ denote any simple path such that $f_{c_{i}}(0)=c_{i}, f_{c_{i}}(1)=0$, and $f_{c_{i}}([0,1])=c_{i} 0$. Also, cut the circle $C_{\theta}$ into two halves by the line $\ell$ that passes through 0 and the center $c$ of $C_{\theta}$. Define $C_{\theta}^{1}\left(C_{\theta}^{2}\right.$, respectively) to be one of the halves that contains $c_{1}$ and $R_{a}\left(c_{2}\right)$ ( $c_{2}$ and $R_{a}\left(c_{1}\right)$, respectively).

Lemma 3.13. For $i=1,2$, the path $R_{a} \circ f_{c_{i}}:[0,1] \rightarrow C_{\theta}$ is a simple path from $R_{a}\left(c_{i}\right)$ to 0 , and its image is $R_{a}\left(c_{i}\right) 0$.

Proof. We will prove the statement only for $i=1$. The composite $R_{a} \circ f_{c_{1}}$ is a path from $[0,1]$ into $C_{\theta}$ with the initial point $\left(R_{a} \circ f_{c_{1}}\right)(0)=R_{a}\left(c_{1}\right)$ and the final point $\left(R_{a} \circ f_{c_{1}}\right)(1)=R_{a}(0)=0$. In order to show that $R_{a} \circ f_{c_{1}}$ is a simple path, suppose that it is not. Then there exist $\alpha_{1}$ and $\alpha_{2}$ such that $0 \leq \alpha_{1}<\alpha_{2} \leq 1$ and $\left(R_{a} \circ f_{c_{1}}\right)\left(\alpha_{1}\right)=\left(R_{a} \circ f_{c_{1}}\right)\left(\alpha_{2}\right)$. Since $R_{a} \circ f_{c_{1}}$ is a continuous path with its image in $C_{\theta}$, at least one of the following three subcases must occur.

Subcase 1. There exist $\beta_{1}, \beta_{2}, \beta_{3}$ satisfying either $0 \leq \beta_{1} \leq \alpha_{1}<\beta_{2}<\beta_{3} \leq \alpha_{2} \leq 1$ or $0 \leq \alpha_{1} \leq \beta_{1}<\beta_{2}<\alpha_{2} \leq \beta_{3} \leq 1$, and $\left(R_{a} \circ f_{c_{1}}\right)\left(\beta_{1}\right)=\left(R_{a} \circ f_{c_{1}}\right)\left(\beta_{2}\right)=$ $\left(R_{a} \circ f_{c_{1}}\right)\left(\beta_{3}\right)$. This is a contradiction, since $R_{a}$ is of degree 2 and the pre-images of any point under $R_{a}$ must consist of two points, counting multiplicity. (Here, we use the fact that the path $f_{c_{1}}$ is simple.)

Subcase 2. For $0=\alpha_{1}<\alpha_{2}<1$ we have $\left(R_{a} \circ f_{c_{1}}\right)\left(\alpha_{1}\right)=\left(R_{a} \circ f_{c_{1}}\right)\left(\alpha_{2}\right)=$ $R_{a}\left(c_{1}\right)$. Since $c_{1}$ is a critical point of $R_{a}$, the pre-images of $R_{a}\left(c_{1}\right)$ consist of $c_{1}$ with multiplicity 2. However, the above equation shows that $R_{a}\left(c_{1}\right)$ has three pre-images, $c_{1}, c_{1}$, and $f_{c_{1}}\left(\alpha_{2}\right) \neq c_{1}$, counting multiplicity.

Subcase 3. For $0<\alpha_{1}<\alpha_{2}=1$ we have $\left(R_{a} \circ f_{c_{1}}\right)\left(\alpha_{1}\right)=\left(R_{a} \circ f_{c_{1}}\right)\left(\alpha_{2}\right)=0$. This is a contradiction, since then the pre-images of 0 under $R_{a}$ are 0,1 , and $f_{c_{1}}\left(\alpha_{1}\right)$, the last of which is neither 0 nor 1 by Remarks 3.10 . 
Thus, $R_{a} \circ f_{c_{1}}$ is a simple path. Note that part of the image of $f_{c_{1}},\left\{f_{c_{1}}(x)\right.$ : $1-\epsilon<x<1\}$ for small enough $\epsilon>0$, is in $C_{\theta}^{1}$ and is contained in a petal at 0 . This portion is mapped by $R_{a}$ to a Fatou component that contains the other petal at 0 , so the image of this portion must lie on $C_{\theta}^{2}$. Thus the image of the path $R_{a} \circ f_{c_{1}}$ must coincide with $R_{a}\left(c_{1}\right) 0$.

Similarly, by cutting the circle $C_{\theta}$ at $c_{i}$ and 1 , we form on $C_{\theta}$ two $\operatorname{arcs}$ whose end points are $c_{i}$ and 1 . Define $c_{i} 1$ to be the arc that does not intersect with $c_{i} 0$ except at $c_{i}$. Let $g_{c_{i}}:[0,1] \rightarrow \mathbb{C}$ denote any simple path such that $g_{c_{i}}(0)=c_{i}$, $g_{c_{i}}(1)=1$, and $g_{c_{i}}([0,1])=c_{i} 1$. Techniques in the proof of Lemma 3.13 can be applied to obtain the following.

Lemma 3.14. For $i=1,2$, the path $R_{a} \circ g_{c_{i}}:[0,1] \rightarrow C_{\theta}$ is a simple path from $R_{a}\left(c_{i}\right)$ to 0 , and its image is $R_{a}\left(c_{i}\right) 0$.

Using these paths we now show that critical orbits converge to 0 .

Proposition 3.15. The orbit of $c_{1}$ converges to 0 for $\theta \in\left(\frac{\pi}{2}, \pi\right)$.

Proof. The simple path $f_{c_{1}}$ has image $c_{1} 0$. Lemma 3.13 states that the composite $R_{a} \circ f_{c_{1}}$ is a simple path with image $R_{a}\left(c_{1}\right) 0$. Lemma 3.11 implies that $R_{a}\left(c_{1}\right) 0 \subset$ $c_{2} 0$. In turn, the simple path $f_{c_{2}}$ has image $c_{2} 0$, and Lemma 3.13 states that the composite $R_{a} \circ f_{c_{2}}$ is a simple path with image $R_{a}\left(c_{2}\right) 0$, which lies on $C_{\theta}^{1}$. Hence $R_{a}\left(c_{1}\right) 0$ must be mapped to $R_{a}{ }^{2}\left(c_{1}\right) 0$. Since $\left|R_{a}{ }^{2}\left(c_{1}\right)\right|<\left|c_{1}\right|$ by Lemma 3.12, the inclusion $R_{a}^{2}\left(c_{1}\right) 0 \subset c_{1} 0$ holds. Inductively, we obtain the following sequences:

$$
\begin{aligned}
& \cdots \subset R_{a}{ }^{2(n+1)}\left(c_{1}\right) 0 \subset R_{a}{ }^{2 n}\left(c_{1}\right) 0 \subset R_{a}{ }^{2(n-1)}\left(c_{1}\right) 0 \subset \cdots \subset R_{a}{ }^{2}\left(c_{1}\right) 0 \subset c_{1} 0 ; \\
& \cdots \subset R_{a}{ }^{2 n+1}\left(c_{1}\right) 0 \subset R_{a}{ }^{2 n-1}\left(c_{1}\right) 0 \subset R_{a}{ }^{2 n-3}\left(c_{1}\right) 0 \subset \cdots \subset R_{a}\left(c_{1}\right) 0 \subset c_{2} 0 .
\end{aligned}
$$

From these we obtain two monotone decreasing sequences, $\left\{\left|R_{a}{ }^{2 n}\left(c_{1}\right)\right|\right\}$ and $\left\{\left|R_{a}{ }^{2 n-1}\left(c_{1}\right)\right|\right\}$. By Lemma 3.13 both sequences have only positive terms. The Monotone Sequence Theorem implies that both sequences converge. Suppose that $\lim _{n \rightarrow \infty}\left|R_{a}{ }^{2 n}\left(c_{1}\right)\right|=\alpha$. Since the points $R_{a}{ }^{2 n}\left(c_{1}\right)$ lie on $c_{1} 0$ and $c_{1} 0$ is contained in $C_{\theta}^{1}$, which is half of a circle having 0 as one of its end points, $c_{1} 0$ and the circle of center 0 and radius $\alpha$ have only one intersection $A$. Then it must be $\lim _{n \rightarrow \infty} R_{a}^{2 n}\left(c_{1}\right)=A$.

We claim that $\lim _{n \rightarrow \infty} R_{a}^{2 n}\left(c_{1}\right)=0$. To this end, note that from the continuity of $R_{a}$ we have

$$
A=\lim _{n \rightarrow \infty} R_{a}^{2(n+1)}\left(c_{1}\right)=R_{a}^{2}\left(\lim _{n \rightarrow \infty} R_{a}^{2 n}\left(c_{1}\right)\right)=R_{a}{ }^{2}(A) .
$$

Hence $R_{a}{ }^{2}(A)=A$. Since $R_{a}{ }^{2}$ fixes $0, \infty$, and $\frac{2}{1-a}, A$ must be one of these. Here, $\infty$ is inappropriate since $A$ lies on the circle $C_{\theta}$ in $\mathbb{C}$. Also, $\frac{2}{1-a}$ is inappropriate since it is the center of $C_{\theta}$. Thus, $A$ must be 0 . A similar argument shows that $\lim _{n \rightarrow \infty} R_{a}{ }^{2 n-1}\left(c_{1}\right)=0$. This completes the proof of Proposition 3.15.

Proposition 3.16. The orbit of $c_{2}$ converges to 0 for $\theta \in\left(\frac{\pi}{2}, \pi\right)$.

Proof. Unlike $c_{1}$, for which Lemma 3.11 showed that $\left|R_{a}\left(c_{1}\right)\right|<\left|c_{2}\right|$ holds for $\theta \in\left(\frac{\pi}{2}, \pi\right)$, a similar inequality for $c_{2}$ does not hold for all $\theta \in\left(\frac{\pi}{2}, \pi\right)$. Instead, when $\theta=\cos ^{-1}\left(-\frac{3}{4}\right), c_{1}$ and $R_{a}\left(c_{2}\right)$ coincide, and depending on the value of $\theta$ we have either

(i) $\left|R_{a}\left(c_{2}\right)\right|<\left|c_{1}\right|$ for $\theta \in\left(\cos ^{-1}\left(-\frac{3}{4}\right), \pi\right)$ or 
(ii) $\left|R_{a}\left(c_{2}\right)\right|>\left|c_{1}\right|$ for $\theta \in\left(\frac{\pi}{2}, \cos ^{-1}\left(-\frac{3}{4}\right)\right)$.

For (i) an argument analogous to the one in Proposition 3.15 shows that the orbit of $c_{2}$ converges to 0 . For (ii) we observe the following. For a simple path $f_{c_{2}}$ from $c_{2}$ to 0 with image $c_{2} 0$, the composite $R_{a} \circ f_{c_{2}}$ is a simple path with image $R_{a}\left(c_{2}\right) 0$ that contains $c_{1}$. Lemmas 3.13 and 3.14 with Remarks 3.10 imply that $R_{a}{ }^{2} \circ f_{c_{2}}$ is a concatenation of two simple paths, one from $R_{a}{ }^{2}\left(c_{2}\right)$ to $R_{a}\left(c_{1}\right)$ and the other from $R_{a}\left(c_{1}\right)$ to 0 . Using Lemma 3.11 we obtain

$$
R_{a}{ }^{2}\left(c_{2}\right) 0 \subset R_{a}\left(c_{1}\right) 0 \subset c_{2} 0 .
$$

The composite $R_{a} \circ f_{c_{2}}$ is a simple path. This, coupled with the proof of Proposition 3.15 showing that $R_{a}^{2}\left(c_{1}\right) 0 \subset c_{1} 0$, gives

$$
R_{a}{ }^{3}\left(c_{2}\right) 0 \subset R_{a}{ }^{2}\left(c_{1}\right) 0 \subset c_{1} 0 .
$$

By induction we obtain

$$
R_{a}{ }^{n+1}\left(c_{2}\right) 0 \subset R_{a}^{n}\left(c_{1}\right) 0 .
$$

Since $\lim _{n \rightarrow \infty} R_{a}{ }^{n}\left(c_{1}\right)=0, \lim _{n \rightarrow \infty} R_{a}{ }^{n}\left(c_{2}\right)=0$ also. Lastly, for $\theta=\cos ^{-1}\left(-\frac{3}{4}\right)$ we have $\left|R_{a}\left(c_{2}\right)\right|=\left|c_{1}\right|$ and hence $R_{a}\left(c_{2}\right)=c_{1}$; thus both critical orbits converge to the parabolic fixed point 0 .

This completes the proof of Theorem 3.1

We have additional results on the Fatou and Julia sets of $R_{a}$. For a rational map $R$ on $\widehat{\mathbb{C}}$, the Fatou set $F(R)$ of $R$ is the maximal open subset of $\widehat{\mathbb{C}}$ in which $\left\{R^{n}\right\}_{n \in \mathbb{N}}$ forms a normal family. The Julia set $J(R)$ is the complement of the Fatou set. The Fatou set $F$ and the Julia set $J$ are completely invariant under $R: R^{-1}(F)=F=R(F)$ and $R^{-1}(J)=J=R(J)$. Also, for any positive integer $n, F\left(R^{n}\right)=F(R)$ and $J\left(R^{n}\right)=J(R)$. If $\operatorname{deg} R \geq 2$, then $J(R)$ is a perfect set, and either $J=\widehat{\mathbb{C}}$ or $J$ has empty interior ([2]). The following corollary is an easy consequence of Case 4.

Corollary 3.17. For $a \in A_{4}$, points on $C_{\theta} \backslash\{0,1\}$ are in the Fatou set of $R_{a}$.

For each $a$ in $A_{2}$, we can show that the Julia set $J$ of $R_{a}$ is a Jordan curve. In fact, we have the following result.

Proposition 3.18. For $a \in A_{2}, J\left(R_{a}\right)=C_{\mathbb{R}}=\{z \in \mathbb{R}\} \cup\{\infty\}$.

Proof. The circle $C_{\mathbb{R}}$ is completely invariant under $R_{a}$. By the minimality of the Julia set, $C_{\mathbb{R}} \supset J$. The set $J$ cannot be totally disconnected since $R_{a}$ has a parabolic fixed point whose immediate basin consists of disjoint Fatou components. If $C_{\mathbb{R}} \neq J$, then both $J$ and the closure of $C_{\mathbb{R}} \backslash J$ are infinite and completely invariant. However, this cannot happen by the minimality of $J$.

\section{Comparison with the Mandelbrot set}

In this section we define and explain equivalent statements for the Mandelbrot set $M$. Using Theorem 3.1 we define a Mandelbrot-set-like object $M^{\prime}$ for $\left\{R_{a}\right\}$ and observe differences in dynamical properties between $M$ and $M^{\prime}$ that the existence of a parabolic fixed point brings about. Let $P_{c}(z)=z^{2}+c$. For all $c \in \mathbb{C}$, the quadratic polynomial $P_{c}$ has a super-attracting fixed (hence critical) point $\infty$ and 
one critical point 0 in $\mathbb{C}$. In the dynamical plane of $P_{c}$, the immediate basin $A_{\infty}$ of $\infty$ is both forward and backward invariant under $P_{c}$; in particular, $A_{\infty}=\{z \in$ $\left.\widehat{\mathbb{C}}: P_{c}{ }^{n}(z) \rightarrow \infty\right\}$. The complement $\widehat{\mathbb{C}} \backslash A_{\infty}=\left\{z \in \mathbb{C}: P_{c}{ }^{n}(z) \nrightarrow \infty\right\}$ is bounded and hence compact. The boundary of $A_{\infty}$ is the Julia set of $P_{c}$.

The parameter space of $\left\{P_{c}\right\}$ depicts the Mandelbrot set $M$. The Mandelbrot set has two, possibly three, equivalent characterizations.

Definition A. The Mandelbrot set $M$ is defined by $M=\left\{c \in \mathbb{C}: P_{c}{ }^{n}(0) \nrightarrow \infty\right\}$.

Proposition B. The Mandelbrot set is the connectivity locus of $J\left(P_{c}\right)$.

Conjecture C. The Mandelbrot set is the closure of the hyperbolicity locus of $P_{c}$.

Proposition D. The Mandelbrot set is the "filled" bifurcation locus of $\left\{P_{c}\right\}$.

Definition A means that the Mandelbrot set is the set of $c$-values for which the critical point 0 of $P_{c}$ is not in $A_{\infty}$ and hence the critical orbit is bounded.

Proposition B means that the Mandelbrot set $M$ is equivalent to the set of $c$ values for which the Julia set of $P_{c}=z^{2}+c$ is connected. This equivalence goes back to Fatou and Julia (see e.g. Branner [4]). Fatou and Julia proved that $J\left(P_{c}\right)$ is connected if and only if the critical point 0 of $P_{c}$ has bounded orbit. Moreover, they showed that there is a complete dichotomy of the parameter space of $\left\{P_{c}\right\}$ : $J\left(P_{c}\right)$ is either connected (when $c \in M$ ) or totally disconnected $\left(c \in M^{c}\right.$ ).

With Definition A, any $c$ for which $P_{c}$ has an attracting cycle other than $\infty$ lies in the Mandelbrot set $M$. A rational map is hyperbolic if every critical point of it is associated to an attracting cycle. It is conjectured that $M$ is the topological closure of the set of hyperbolic parameters for $P_{c}$, which leads to Conjecture C.

To explain Proposition D, we recall (see e.g. Keen [8]) that given a map $f_{x}$ in a parameterized family of holomorphic rational maps $\left\{f_{\lambda}\right\}$, the Julia set $J\left(f_{x}\right)$ moves holomorphically if for all $x^{\prime}$ in some neighborhood of $x, f_{x}$ is quasiconformally conjugate to $f_{x^{\prime}}$. The set of $\lambda$-values for which $J\left(f_{\lambda}\right)$ moves holomorphically is called the $J$-stable set, and its complement the bifurcation locus. The maps enjoy a type of structural stability as the parameter $\lambda$ varies in a component of the $J$-stable set. For example, the number of attracting cycles and the period of each of them remain locally constant for maps corresponding to parameters in a component of the $J$-stable set (McMullen [11). Mañé, Sad, and Sullivan [10 showed that the $J$-stable set is open and dense in the parameter space. The set of $\lambda$-values for which $f_{\lambda}$ is hyperbolic forms an open and closed subset of the $J$-stable set ([11]). A component $U$ is hyperbolic if for some parameter $\lambda$ in $U$, and hence for all $\lambda$ in $U, f_{\lambda}$ is hyperbolic.

With Definition A, McMullen 11 proved that the boundary of the Mandelbrot set is the bifurcation locus of $\left\{P_{c}\right\}$, thus obtaining Proposition D. Moreover, he proved the following result.

Theorem 4.1 (12). Quasiconformal copies of $\partial M$ or a generalized version are dense in the bifurcation locus of any parameterized family of holomorphic rational maps.

Now we compare $\left\{P_{c}\right\}$ and $\left\{R_{a}\right\}$. For $\left\{P_{c}\right\}$, each map has two critical points. The "pre-determined" critical point $\infty$ itself is a super-attracting fixed point. The Mandelbrot set $M$ is defined as the set of $c$-values for which the orbit of the "free" 


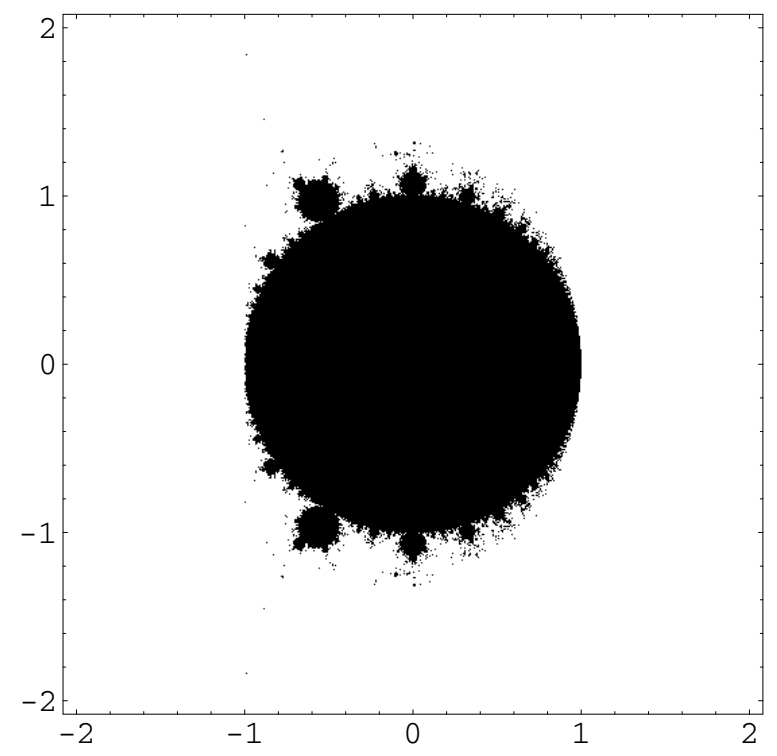

FiguRE $4 . M^{\prime}$.

critical point 0 does not converge to the "pre-determined" critical point $\infty: M=$ $\left\{c \in \mathbb{C}: P_{c}^{n}(0) \nrightarrow \infty\right\}$. Also, the parameter space of $\left\{P_{c}\right\}$ is already reduced; i.e., no two members of $\left\{P_{c}\right\}$ are conformally conjugate to each other.

As for $\left\{R_{a}\right\}$, each map has two distinct critical points $c_{1}$ and $c_{2}$. Assuming that $c_{1}$ is associated to the parabolic fixed point 0 for all parameters in the reduced set $\mathcal{R}_{a}$ (and thus assuming a stronger statement than Theorem 3.1), $c_{1}$ is the "pre-determined" critical point, and the behavior of the "free" critical point $c_{2}$ determines some features of $\mathcal{R}_{a}$. This shows similarities between the two families $\left\{P_{c}\right\}$ and $\left\{R_{a}\right\}$. We are interested in characterizing $\mathcal{R}_{a}$.

Definition (A). Define a set $M^{\prime}$ by $M^{\prime}=\left\{a \in \mathcal{R}_{a}: R_{a}{ }^{n}\left(c_{2}\right) \nrightarrow 0\right\}$.

The set $M^{\prime}$ is the set of $a$-values for which the orbit of the "free" critical point $c_{2}$ does not converge to the destination 0 of the orbit of the "pre-determined" critical point $c_{1}$. Figure 4 shows $M^{\prime}$ in $[-2,2] \times[-2 i, 2 i]$.

The following results state that the reduced set $\mathcal{R}_{a}$ cannot be characterized as depicting the connectivity locus or hyperbolicity locus of $\left\{R_{a}\right\}$.

Theorem 4.2 ([16]). If a quadratic rational map $R$ has an invariant Fatou component containing two critical points, then the Julia set $J(R)$ is a Cantor set. Otherwise, $J(R)$ is connected.

Corollary (B). The Julia set $J\left(R_{a}\right)$ is connected for all $a \in \mathcal{R}_{a}$.

Proof. Theorem 4.2 implies that the Julia set of a quadratic rational map $R$ is either connected or totally disconnected. If $J(R)$ is totally disconnected, then its complement $F(R)$ is connected and consists of a single component. By construction $R_{a}$ has a parabolic fixed point whose immediate basin consists of two disjoint Fatou components. Hence the Julia set $J\left(R_{a}\right)$ cannot be totally disconnected. Thus $J\left(R_{a}\right)$ is connected for all $a \in \mathcal{R}_{a}$. 
Proposition (C). The map $R_{a}$ is not hyperbolic for any $a \in \mathcal{R}_{a}$.

Proof. By construction $R_{a}$ always has a parabolic fixed point, and a critical point must be associated to it. Hence the map $R_{a}$ is not hyperbolic for any $a \in \mathcal{R}_{a}$.

Thus, describing the reduced set $\mathcal{R}_{a}$ as depicting the bifurcation locus of $\left\{R_{a}\right\}$ seems most appropriate.

Conjecture (D). The bifurcation locus of $\left\{R_{a}\right\}$ is $\partial M^{\prime}$.

With Theorem 4.1 and Definition (A) we form the following conjecture.

Conjecture (E) (cf. 13]). The bifurcation locus of $\left\{R_{a}\right\}$ is a quasiconformal image of the boundary of the Mandelbrot set without the period 2 limb.

\section{APPENDiX: COMPUTER ALGORITHM FOR DRAWING THE PARAMETER SPACE OF $\left\{R_{a}\right\}$}

We explain the algorithm that produces the parameter space picture of $\left\{R_{a}\right\}$. The algorithm basically tracks critical orbits.

Computer algorithm for drawing the parameter space of $\left\{R_{a}\right\}$.

(i) Each pixel represents a parameter value $a$ corresponding to the map $R_{a}$. Assign the default value 0 to each $a$.

(ii) For each $a$, we iterate the critical points $N>0$ times to start. Let $s_{i}=$ $R_{a}{ }^{N}\left(c_{i}\right)$.

(iii) Choose $\epsilon>0$ and set $M \in \mathbb{N}$ to be the maximal number of iterations. Apply the following test for each $c_{i}$ : If the inequality $\left|R_{a}{ }^{n}\left(s_{i}\right)-s_{i}\right|<\epsilon$ is satisfied in either the Euclidean metric or the spherical metric for some $n \in \mathbb{N}$, where $1 \leq n \leq M$, then we assign the value 1 to $a$.

(iv) Assign to each $a$ the number of times the value 1 is assigned in (iii). Shade the parameters according to these numbers so that the parameters with the largest number are colored white and those with the smallest number are colored black.

The algorithm is supported by the following reasons. Generally speaking, if an attracting cycle has a multiplier of small modulus, then the iterates of the associated critical point(s) in the attracting immediate basin approach the points in the cycle quite quickly. This enables the inequality in (iii) to hold. Also, if a critical point is associated to a parabolic cycle, then although the attraction is very, very slow so that we may have to take many iterations (by letting $N$ and $n$ be large), the inequality in (iii) eventually holds. On the other hand, if a critical point is associated to a cycle of Siegel disks, then the image of the critical point after a finite number of iterations may not return near the critical point itself. This results in the inequality in (iii) being false for most $\epsilon>0$ and most $n$.

\section{REFERENCES}

[1] Baker, I.N. Fixpoints of polynomials and rational functions. J. London Math. Soc. 39 (1964), 615-622. MR0169989 (30:230)

[2] Beardon, A.F. Iteration of Rational Functions. Springer-Verlag, 1991. MR1128089 (92j:30026)

[3] Bergweiler, W. On the number of critical points in parabolic basins. Ergod. Th. \& Dynam. Sys. 22 (2002), 655-669. MR1908548 (2003k:37059) 
[4] Branner, B. The Mandelbrot set. In Chaos and Fractals, Proc. Sympos. Appl. Math. 39, pages 75-105. American Math. Soc., 1989. MR 1010237

[5] Buff, X., and Epstein, A. A parabolic Pommerenke-Levin-Yoccoz inequality. Fundamenta Mathematicae 172 (2002), 249-289. MR 1898687 (2003b:37067)

[6] Hagihara, R. Rational Maps Lacking Certain Periodic Orbits. PhD thesis, University of North Carolina at Chapel Hill, 2007.

[7] Hawkins, J. Lebesgue ergodic rational maps in parameter space. Int. J. Bifurcation and Chaos 13 (2003), no. 6, 1423-1447. MR1992056 (2004e:37065)

[8] Keen, L. Julia sets of rational maps. In Complex Dynamical Systems: The Mathematics behind the Mandelbrot and Julia Sets, Proc. Sympos. Appl. Math. 49, pages 71-89. American Math. Soc., 1994. MR1315534

[9] Kisaka, M. On some exceptional rational maps. Proc. Japan Acad., Ser. A 71 (1995), 35-38. MR.1326795 (96a:30029)

[10] Mañé, R., Sad, P., and Sullivan, D. On the dynamics of rational maps. Ann. Scient. École Norm. Sup., $4^{\mathrm{e}}$ série 16 (1983), 193-217. MR732343(85j:58089)

[11] McMullen, C. Complex Dynamics and Renormalization. Annals of Math. Studies 135, Princeton Univ. Press, 1994. MR1312365 (96b:58097)

[12] McMullen, C. The Mandelbrot set is universal. In The Mandelbrot Set, Theme and Variations, London Math. Soc. Lecture Note Series 274, pages 1-17. Cambridge University Press, 2000. MR:1765082 (2002f:37081)

[13] Milnor, J. Geometry and dynamics of quadratic rational maps. Experiment. Math. 2 (1993), no. 1, 37-83. MR 1246482 (96b:58094)

[14] Milnor, J. On rational maps with two critical points. Experiment. Math. 9 (2000), no. 4, 481-522. MR 1806289 (2001k:37074)

[15] Rees, M. Components of degree two hyperbolic rational maps. Invent. Math. 100 (1990), no. 2, 357-382. MR:1047139 (91b:58187)

[16] Yin, Y.-C. On the Julia sets of quadratic rational maps. Compl. Variab. 18 (1992), 141-147. MR:1157922 (93e:58160)

School of Mathematics and Statistics, University of New South Wales, Sydney, NSW 2052, Australia

E-mail address: r.hagihara@unsw.edu.au 